\title{
The Generalization of the Poisson Sum Formula Associated with the Linear Canonical Transform
}

\author{
Jun-Fang Zhang and Shou-Ping Hou \\ Department of Basic Science, Beijing University of Agriculture, Beijing 102206, China \\ Correspondence should be addressed to Jun-Fang Zhang, zhangjunfang@bac.edu.cn
}

Received 1 August 2012; Revised 24 November 2012; Accepted 24 November 2012

Academic Editor: Baolin Wang

Copyright (C) 2012 J.-F. Zhang and S.-P. Hou. This is an open access article distributed under the Creative Commons Attribution License, which permits unrestricted use, distribution, and reproduction in any medium, provided the original work is properly cited.

The generalization of the classical Poisson sum formula, by replacing the ordinary Fourier transform by the canonical transformation, has been derived in the linear canonical transform sense. Firstly, a new sum formula of Chirp-periodic property has been introduced, and then the relationship between this new sum and the original signal is derived. Secondly, the generalization of the classical Poisson sum formula to the linear canonical transform sense has been obtained.

\section{Introductions}

As a generalization of the classical Fourier transform and the fractional Fourier transform (FrFT), the linear canonical transform (LCT) receives much interest in recent years [1-3]. Many important transforms, for example, the Fourier transform, the Fresnel transform, and the scaling operations are all special cases of the LCT. It has been shown to be one of the most useful tools in several areas [4-6], including optics, quantum physics, and in the signal processing community. Its relationship with the Fourier transform and the fractional Fourier transform can be found in $[7,8]$. The well-known operations, for example, the Hilbert transform, the Parseval relationship, the convolution and product operations, and the spectral analysis, in traditional Fourier domain have been extended to the linear canonical transform domain by different authors [9-13]. For further properties and applications of LCT in optics and signal processing community, one can refer to $[1,2]$. The classical sampling theorems associated with the LCT have also been investigated and studied in the LCT domain in various literatures. The extensions of the classical Shannon sampling theorem for bandlimited or time-limited signals in the LCT domain have been deduced in [13, 14].

However, for the best of our knowledge, none of the research papers throw light on the study of the traditional Poisson sum formula [15-21] associated with the LCT have been 
reported as yet. The Poisson summation formula is a very useful tool not only in many branches of the mathematics, but also it finds many applications in various fields, for example, mechanics, signal processing community, and many scientific fields. It is therefore, worthwhile as well as interesting to investigate the Poisson sum formula associated with the LCT.

The objective of this paper is to study and investigate the Poisson formula associated with the LCT. In other words, we want to generalize the classical Poisson sum formula by replacing the ordinary Fourier transform by the canonical transform. In order to obtain the desired results for a signal $x(t)$, we first deduce a new sum formula for the signal $x(t)$ and then achieve the innovative results in the LCT domain. The paper is organized as follows, the preliminaries are proposed in Section 2, the main results of the paper are investigated in Section 3, and the conclusion is given in Section 4.

\section{Preliminaries}

\subsection{The Linear Canonical Transform}

The linear canonical transform (LCT) of a signal $x(t)$ with parameter matrix $A$ is defined as $[1-3]$

$$
X_{A}(u)=\int_{-\infty}^{\infty} K_{A}(u, t) x(t) d t
$$

where

$$
K_{A}(u, t)=\sqrt{\frac{1}{j 2 \pi b}} e^{j\left((a / 2 b) t^{2}-(1 / b) u t+(d / 2 b) u^{2}\right)}, \quad b \neq 0,
$$

and $X_{A}(u)=\sqrt{d} e^{j(c d / 2) u^{2}} x(d u), b=0$. The parameter $A=\left(\begin{array}{cc}a & b \\ c & d\end{array}\right)$, and satisfy $\operatorname{det}(A)=1, a$, $b, c, d \in R$. In this case, the linear canonical transform is a unitary transform [1], therefore we can derive the inverse transform of LCT as another LCT transform.

The inverse transform of LCT can be derived by an LCT with the parameter of $A^{-1}$ as following:

$$
x(t)=\int_{-\infty}^{\infty} K_{A^{-1}}(t, u) X_{A}(u) d u .
$$

The LCT can be looked at as the generalization of the well-known operations in the science and engineering community [4-6]. The relationship between the LCT and the Fourier transform, the fractional Fourier transform have been derived in [1-3].

A signal $x(t)$ is said to be band-limited with respect to $\Omega_{A}$ in linear canonical transform domain, when

$$
X_{A}(u)=0 \text { for }|u|>\Omega_{A}
$$


where $\Omega_{A}$ is called the bandwidth of signal $x(t)$ in the linear canonical transform domain. At the same time, a signal $x(t)$ is called Chirp-periodic with period $T$ and of parameter $A$ if it satisfies the following equation:

$$
e^{j\left(\left(a t^{2}\right) / 2 b\right)} x(t)=e^{j\left(a(t+T)^{2} / 2 b\right)} x(t+T) .
$$

The following identities will be used in the following sections.

Lemma 2.1. The inverse linear canonical transform of signal $Z_{A}(u)=\sum_{n=-\infty}^{+\infty} \delta(u-n B) e^{j(a / 2 b) u^{2}}$ for parameter $A=\left(\begin{array}{ll}a & b \\ c & d\end{array}\right)$ is

$$
z(t)=\frac{1}{B} \sqrt{\frac{-1}{j 2 \pi}} e^{-j(a / 2 b) t^{2}} \sum_{n=-\infty}^{\infty} \delta\left(\frac{t}{b}-\frac{n}{B}\right) .
$$

And the inverse linear canonical transform of signal $\delta\left(u-u_{0}\right)$ is

$$
s(t)=\sqrt{\frac{-1}{j 2 \pi b}} e^{-j(a / 2 b)\left(t^{2}+u_{0}^{2}\right)+j u_{0} t b} .
$$

Proof. These results can be derived easily by the definition of the LCT and the inverse transform of LCT.

Assuming a signal $x(t)$ is band-limited to $\Omega_{A}$ in the linear canonical transform domain, then from the results derived in [13], $x(t)$ is not band-limited in the traditional Fourier domain. Therefore, the classical results of bandlimited signal processing method in Fourier domain can be used in the LCT domain to obtain the novel results associated with the LCT.

\subsection{The Poisson Sum Formula}

The Poisson sum formula demonstrates that the sum of infinite samples in time domain of a signal $x(t)$ is equivalent to the sum of infinite samples of $X(u)$ in the Fourier domain. Mathematically, the Poisson sum formula can be represented as follows:

$$
\sum_{n=-\infty}^{\infty} x(t+k \tau)=\frac{1}{\tau} \sum_{n=-\infty}^{\infty} X\left(\frac{n}{\tau}\right) e^{j(n / \tau) t}
$$

or

$$
\sum_{k=-\infty}^{\infty} x(k \tau)=\frac{1}{\tau} \sum_{n=-\infty}^{\infty} X\left(\frac{n}{\tau}\right)
$$

where $X(u)$ is the traditional Fourier transform of signal $x(t)$. It is well known that it will be valid only if $x(t)$ and its Fourier transform $X(u)$ are regular enough and only if both series of (2.9) converge [20]. 
In order to obtain the new results associated with the linear canonical transform, a new summation associated with the signal $x(t)$ is introduced as following:

$$
y(t)=\sum_{k=-\infty}^{\infty} x(t+k \tau) e^{j(a / 2 b)\left(2 k \tau t+k^{2} \tau^{2}\right)},
$$

where $\tau$ is a constant. From (2.10), the function $y(t)$ can be seen as a periodic phase-shift replica of the original function $x(t)$. The signal $y(t)$ will be used in the following sections to investigate the Poisson sum formula associate with the linear canonical transform.

\section{The Main Results}

Suppose a signal $x(t)$ is band-limited to $\Omega_{A}$ in linear canonical transform domain of parameter $A$, then, from (2.10) a new function $y(t)$ can be deduced from signal $x(t)$. Firstly, the properties of the signal $y(t)$ associated with the linear canonical transform can be derived from Theorem 3.1.

Theorem 3.1. Suppose a signal $x(t)$ is band-limited to $\Omega_{A}$ in the linear canonical transform domain of parameter $A$, and $y(t)=\sum_{k=-\infty}^{\infty} x(t+k \tau) e^{j(a / 2 b)\left(2 k \tau t+k^{2} \tau^{2}\right)}$, then the following results about $y(t)$ is true.

(a) $y(t)$ is a Chirp-periodic signal with period $\tau$.

(b) $x(t)$ is a band-limited signal in linear canonical transform domain with parameter $A$, if and only if $y(t)$ has a finite number of nonzero linear canonical series coefficients for any $\tau$.

Proof. (a) By the definition of the Chirp-periodicity, we obtain

$$
\begin{aligned}
y(t+\tau) e^{j(a / 2 b)(t+\tau)^{2}} & =\sum_{k=-\infty}^{\infty} x(t+\tau+k \tau) e^{j(a / 2 b)\left[2 k \tau(t+\tau)+k^{2} \tau^{2}\right]} e^{j(a / 2 b)(t+\tau)^{2}} \\
& =e^{j(a / 2 b) t^{2}} \sum_{k=-\infty}^{\infty} x[t+(k+1) \tau] e^{j(a / 2 b)\left[2(k+1) \tau t+(k+1)^{2} \tau^{2}\right]} \\
& =y(t) e^{j(a / 2 b) t^{2}} .
\end{aligned}
$$

This proves the Chirp-periodicity of the signal $y(t)$.

(b) To prove the necessary condition, the $n$th coefficient $c_{n, A}$ of signal $y(t)$ can be deduced from the linear canonical series definition proposed in [4] as

$$
c_{n, A}=\sqrt{\frac{(b-j a)}{\tau}} \int_{0}^{\tau} y(t) e^{j(a / 2 b)\left[(n b 2 \pi / \tau)^{2}+t^{2}\right]-j(n 2 \pi / \tau) t} d t .
$$


Equation (3.2) can be rewritten as

$$
\begin{aligned}
c_{n, A} & =\sqrt{\frac{(b-j a)}{\tau}} e^{j(a / 2 b)(n 2 \pi b / \tau)^{2}} \int_{0}^{\tau} \sum_{k=-\infty}^{\infty} x(t+k \tau) e^{j(a / 2 b)\left(2 k \tau t+k^{2} \tau^{2}\right)} e^{j(a / 2 b) t^{2}-j(n(2 \pi / \tau)) t} d t \\
& =\sqrt{\frac{(b-j a)}{\tau}} e^{j(a / 2 b)(n 2 \pi b / \tau)^{2}} \sum_{k=-\infty}^{+\infty} \int_{k \tau}^{(k+1) \tau} x(\lambda) e^{j(a / 2 b) \lambda^{2}-j(n(2 \pi / \tau))(\lambda-k \tau)} d \lambda \\
& =\frac{\sqrt{(b-j a) / \tau}}{K_{A}(u, t)} \int_{-\infty}^{+\infty} K_{A}(u, t) x(\lambda) e^{j(a / 2 b)\left(n F^{\prime}\right)^{2}} e^{j(a / 2 b) \lambda^{2}-j(1 / b)\left(n F^{\prime}\right) \lambda} d \lambda \\
& =\frac{\sqrt{(b-j a) / \tau}}{K_{A}(u, t)} X_{A}\left(n \frac{2 \pi}{\tau} b\right) .
\end{aligned}
$$

Since $x(t)$ is a $\Omega_{A}$ band-limited signal in linear canonical transform domain of parameter $A$, that is to say

$$
X_{A}(u)=\int_{-\infty}^{+\infty} x(t) K_{A}(u, t) e^{j(a / 2 b)\left(t^{2}+u^{2}\right)-j(1 / b) u t} d t=0, \quad|u|>\Omega_{\alpha}
$$

Comparing (3.3) and (3.4), we obtain

$$
c_{n, A}=0, \quad \text { when } n>\frac{\tau \Omega_{A}}{2 \pi b} .
$$

Therefore, the necessary condition is proved.

To prove the sufficient condition, let us assume that $c_{n, A}=0$ for $n>N$, where $N$ is any finite integer. From (3.5), the

$$
X_{A}(u)=\int_{-\infty}^{+\infty} x(t) K_{A}(u, t) d t=0, \quad|u|>\frac{2 \pi b N}{\tau}
$$

Hence, $x(t)$ is band-limited signal having bandwidth as following:

$$
\Omega_{A} \leq \frac{2 \pi b N}{\tau}
$$

This proves the sufficient condition of the theorem. deduced.

Based on the derived results of Theorem 3.1, the following Theorem 3.2 can be

Theorem 3.2. Suppose a signal $x(t)$ is band-limited to $\Omega_{A}$ in the linear canonical transform domain of parameter $A$, and $y(t)=\sum_{k=-\infty}^{\infty} x(t+k \tau) e^{j(a / 2 b)\left(2 k \tau t+k^{2} \tau^{2}\right)}$ is derived by shifting signal $x(t)$ to left and right, then the following conclusions can be deduced. 
(a) When $1 / \tau>\Omega_{A}, y(t)$ can be deduced from the following formula:

$$
y(t)=\left(\frac{b}{\tau}\right) X_{A}(0) e^{-j(a / 2 b) t^{2}}
$$

(b) When $\Omega_{A} / 2<1 / \tau<\Omega_{A}, y(t)$ can be deduced from the following formula:

$$
y(t)=\frac{b}{\tau} e^{-j(a / 2 b) t^{2}}\left\{X_{A}(0)+e^{-j(a / 2 b)(b / \tau)^{2}}\left[X_{A}\left(\frac{b}{\tau}\right) e^{j t / \tau}+X_{A}\left(-\frac{b}{\tau}\right) e^{-j t / \tau}\right]\right\}
$$

(c) When $\Omega_{A} / n<1 / \tau<\Omega_{A} /(n-1), y(t)$ can be deduced from the following formula:

$$
y(t)=\frac{b}{\tau} e^{-j(a / 2 b) t^{2}}\left\{X_{A}(0)+\sum_{k=1}^{n} e^{-j(a / 2 b)(k(a / \tau))^{2}}\left[X_{A}\left(k \frac{b}{\tau}\right) e^{j k t / \tau}+X_{A}\left(-k \frac{b}{\tau}\right) e^{-j k t / \tau}\right]\right\}
$$

Proof. (1) Proof of (a)

Since $x(t)$ is a $\Omega_{A}$ band-limited signal in the linear canonical transform domain, $X_{A}(u)$ if sampled in the linear canonical transform domain of order $A$ at a rate of $B>\Omega_{A}$, then the samples can be represented as follows:

$$
Y_{s, A}(u)=X_{A}(u) \sum_{n=-\infty}^{+\infty} \delta(u-n B)=X_{A}(0) \delta(u), \quad B>\Omega_{A}
$$

The first part of (3.11) can be reorganized as

$$
Y_{s, A}(u)=\left[X_{A}(u)\right]\left[\sum_{n=-\infty}^{+\infty} \delta(u-n B) e^{j(a / 2 b) u^{2}}\right] e^{-j(a / 2 b) u^{2}}
$$

If we let $Z_{A}(u)=\sum_{n=-\infty}^{+\infty} \delta(u-n B) e^{j(a / 2 b) u^{2}}$, then (3.12) can be rewritten as

$$
Y_{s, A}(u)=\left[X_{A}(u)\right]\left[Z_{A}(u)\right] e^{-j(a / 2 b) u^{2}} .
$$

Applying the convolution and product theorem proposed in [7] and Lemma 2.1 to (3.13), the inverse linear canonical transform of formula (3.13) can be represented as

$$
\begin{aligned}
y_{s}(t) & =\sqrt{\frac{1}{j 2 \pi b}} e^{-j(a / 2 b) t^{2}}\left(x(t) e^{(a / 2 b) t^{2}} * z(t) e^{j(a / 2 b) t^{2}}\right) \\
& =\sqrt{\frac{1}{j 2 \pi b}} e^{-j(a / 2 b) t^{2}}\left[x(t) e^{j(a / 2 b) t^{2}} * \frac{1}{B} \sum_{n=-\infty}^{+\infty} \delta\left(\frac{1}{b} t-\frac{n}{B}\right)\right] \\
& =\sqrt{\frac{1}{j 2 \pi b}}\left[\frac{1}{B} \sum_{n=-\infty}^{+\infty} x\left(t-\frac{n}{B} b\right) e^{j(a / b)\left[2(n / B) b t-((n / B) b)^{2}\right]}\right] .
\end{aligned}
$$


From the second part of (3.11), the inverse linear canonical transform of $Y_{S, A}(u)$ can be derived as

$$
y_{s}(t)=\sqrt{\frac{1}{j 2 \pi b}} X_{A}(0) e^{-j(a / 2 b) t^{2}}
$$

If we select $\tau=b / B$, then from (3.14)-(3.15)

$$
y(t)=\frac{b}{\tau} \sqrt{\frac{2 \pi}{(1-j(a / b))}} y_{s}(t)=\frac{b}{\tau} X_{A}(0) e^{-j(a / 2 b) t^{2}} .
$$

This proves (a).

(2) Proof of (b)

Similar to the method of proving (a), if $X_{A}(u)$ is sampled in the linear canonical transform domain of parameter $A$ at a rate of $\Omega_{A} / 2<B<\Omega_{A}$, there are essentially three nonzero samples of $X_{A}(u)$ :

$$
Y_{s, A}(u)=X_{A}(u) \sum_{n=-\infty}^{+\infty} \delta(u-n B)=X_{\alpha}(0) \delta(u)+X_{\alpha}(B) \delta(u-B)+X_{\alpha}(-B) \delta(u+B)
$$

In this condition, (3.14) is also correct, and from (3.13), the relationship between $y(t)$ and $y_{s}(t)$ can be derived as follows:

$$
y(t)=B \sqrt{j 2 \pi b} y_{s}(t)
$$

While $y_{s}(t)$ can be deduced from (3.18) and Lemma 2.1 as

$$
\begin{gathered}
y_{s}(t)=\sqrt{1 / j 2 \pi b}\left[X_{A}(0) e^{-j(a / 2 b) t^{2}}+X_{A}(B) e^{-j(a / 2 b)\left(t^{2}+B^{2}\right)+j B t(1 / b)}\right. \\
\left.+X_{A}(-B) e^{-j(a / 2 b)\left(t^{2}+B^{2}\right)-j B t(1 / b)}\right] .
\end{gathered}
$$

If $\tau=b / B$ is chosen, then from (3.18)-(3.19).

$$
\begin{aligned}
y(t) & =B e^{-j(a / 2 b) t^{2}}\left[X_{A}(0)+X_{A}(B) e^{-j(a / 2 b) B^{2}+j B t(1 / b)}+X_{A}(-B) e^{-j(a / 2 b) B^{2}-j B t(1 / b)}\right] \\
& =B e^{-j(a / 2 b) t^{2}}\left\{X_{A}(0)+e^{-j(a / 2 b) B^{2}}\left[X_{A}(B) e^{j B t / b}+X_{A}(-B) e^{-j B t / b}\right]\right\} \\
& =\left(\frac{b}{\tau}\right) e^{-j(a / 2 b) t^{2}}\left\{X_{A}(0)+e^{-j(a / 2 b)(b / \tau)^{2}}\left[X_{A}\left(\frac{b}{\tau}\right) e^{j t / \tau}+X_{A}\left(-\frac{b}{\tau}\right) e^{-j t / \tau}\right]\right\} .
\end{aligned}
$$

Thus, (b) is also proved. 
(3) Proof of (c)

If $X_{A}(u)$ is sampled in linear canonical transform domain of order $A$ at a rate of $\Omega_{A} / n<B<$ $\Omega_{A} /(n-1)$, there are essentially $2 n-1$ nonzero samples remaining

$$
\begin{aligned}
Y_{s, A}(u) & =X_{A}(u) \sum_{n=-\infty}^{+\infty} \delta(u-n B) \\
& =X_{A}(0) \delta(u)+X_{A}(B) \delta(u \pm B)+\cdots+X_{A}[(n-1) B] \delta[u \pm(n-1) B] .
\end{aligned}
$$

Again, (3.14) is also correct in this case, and using similar method in proving (a) and (b), $y(t)$ can be deduced as

$$
\begin{aligned}
y(t)= & B \sqrt{j 2 \pi b} y_{s}(t) \\
=B e^{-j(a / 2 b) t^{2}}[ & X_{A}(0)+X_{A}(B) e^{-j(a / 2 b) B^{2}+j B t / b}+X_{A}(-B) e^{-j(a / 2 b) B^{2}-j B t / b} \\
& \left.\quad+\cdots+X_{A}(n B) e^{-j(a / 2 b)(n B)^{2}+j n B t / b}+X_{A}(-n B) e^{-j(a / 2 b)(n B)^{2}-j n B t / b}\right] \\
= & B e^{-j(a / 2 b) t^{2}}\left\{X_{A}(0)+\sum_{k=1}^{n} e^{-j(a / 2 b)(k B)^{2}}\left[X_{A}(k B) e^{j k B t / b}+X_{A}(-k B) e^{-j k B t / b}\right]\right\} .
\end{aligned}
$$

If we select $\tau=b / B$, then

$$
y(t)=\frac{b}{\tau} e^{-j(a / 2 b) t^{2}}\left\{X_{A}(0)+\sum_{k=1}^{n} e^{-j(a / 2 b)(k(b / \tau))^{2}}\left[X_{A}\left(k \frac{b}{\tau}\right) e^{j k t / \tau}+X_{A}\left(-k \frac{b}{\tau}\right) e^{-j k t / \tau}\right]\right\} .
$$

Part (c) of Theorem 3.1 is proved.

\section{Conclusion}

In this paper, the generalization of the classical Poisson sum formula to the linear canonical transform domain is investigated, by replacing the ordinary Fourier transform by the canonical transform, we firstly derived a new Chirp-periodic sum, and then the classical Poisson summations are generalized to the linear canonical transform domain based on the relationship derived. The classical results can be looked at as the special cases of the derived results. The applications of the derived results in sampling theories, signal analysis will be investigated in the linear canonical transform domain in the future.

\section{Acknowledgments}

This work is supported by National Natural Science Foundations of China (no. 60901058 and no. 61171195) and the Beijing Natural Science Foundation (no.1102029). 


\section{References}

[1] R. Tao, B. Deng, and Y. Wang, Fractional Fourier Transform and Its Applications, Tsinghua University Press, Beijing, China, 2009.

[2] M. Zhu, B.-Z. Li, and G.-F. Yan, "Aliased polyphase sampling associated with the linear canonical transform," IET Signal Processing, vol. 6, no. 6, pp. 594-599, 2012.

[3] K. B. Wolf, Integral Transforms in Science and Engineering, vol. 11 of Mathematical Concepts and Methods in Science and Engineering, Plenum Press, New York, NY, USA, 1979.

[4] C.-P. Li, B.-Z. Li, and T.-Z. Xu, "Approximating bandlimited signals associated with the LCT domain from nonuniform samples at unknown locations," Signal Processing, vol. 92, no. 7, pp. 1658-1664, 2012.

[5] D. Wei, Q. Ran, and Y. Li, "Reconstruction of band-limited signals from multichannel and periodic nonuniform samples in the linear canonical transform domain," Optics Communications, vol. 284, no. 19, pp. 4307-4315, 2011.

[6] W. Qiu, B.-Z. Li, and X.-W. Li, "Speech recovery based on the linear canonical transform," Speech Communication, vol. 50, no. 1, pp. 40-50, 2013.

[7] B. Deng, R. Tao, and Y. Wang, "Convolution theorems for the linear canonical transform and their applications," Science in China. Series F. Information Sciences, vol. 49, no. 5, pp. 592-603, 2006.

[8] S.-C. Pei and J.-J. Ding, "Relations between fractional operations and time-frequency distributions, and their applications," IEEE Transactions on Signal Processing, vol. 49, no. 8, pp. 1638-1655, 2001.

[9] B.-Z. Li and T.-Z. Xu, "Spectral analysis of sampled signals in the linear canonical transform domain," Mathematical Problems in Engineering, vol. 2012, Article ID 536464, 19 pages, 2012.

[10] B.-Z. Li, R. Tao, T.-Z. Xu, and Y. Wang, "The Poisson sum formulae associated with the fractional Fourier transform," Signal Processing, vol. 89, no. 5, pp. 851-856, 2009.

[11] K. K. Sharma and S. D. Joshi, "Signal separation using linear canonical and fractional Fourier transforms," Optics Communications, vol. 265, no. 2, pp. 454-460, 2006.

[12] R. Tao, B.-Z. Li, Y. Wang, and G. K. Aggrey, "On sampling of band-limited signals associated with the linear canonical transform," IEEE Transactions on Signal Processing, vol. 56, no. 11, pp. 5454-5464, 2008.

[13] B.-Z. Li, R. Tao, and Y. Wang, "New sampling formulae related to linear canonical transform," Signal Processing, vol. 87, no. 5, pp. 983-990, 2007.

[14] J. J. Healy and J. T. Sheridan, "Sampling and discretization of the linear canonical transform," Signal Processing, vol. 89, no. 4, pp. 641-648, 2009.

[15] H. G. Feichtinger, "Parseval's relationship for nonuniform samples of signals with several variables," IEEE Transactions on Signal Processing, vol. 40, no. 5, pp. 1262-1263, 1992.

[16] F. Marvasi, "Reflections on the Poisson sum formula and the uniform sampling," IEICE Transactions, vol. e67, no. 9, pp. 494-501.

[17] A. Córdoba, "La formule sommatoire de Poisson," Comptes Rendus des Séances de l'Académie des Sciences. Série I. Mathématique, vol. 306, no. 8, pp. 373-376, 1988.

[18] A. Córdoba, "Dirac combs," Letters in Mathematical Physics, vol. 17, no. 3, pp. 191-196, 1989.

[19] J.-P. Kahane and P.-G. Lemarié-Rieusset, "Remarques sur la formule sommatoire de Poisson," Studia Mathematica, vol. 109, no. 3, pp. 303-316, 1994.

[20] A. L. Durán, R. Estrada, and R. P. Kanwal, "Extensions of the Poisson summation formula," Journal of Mathematical Analysis and Applications, vol. 218, no. 2, pp. 581-606, 1998.

[21] K. Gröchenig, "An uncertainty principle related to the Poisson summation formula," Studia Mathematica, vol. 121, no. 1, pp. 87-104, 1996. 


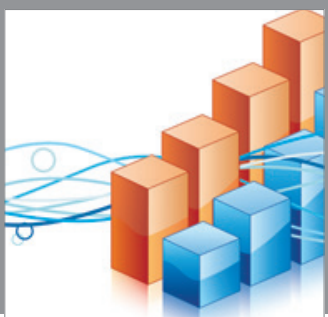

Advances in

Operations Research

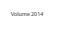

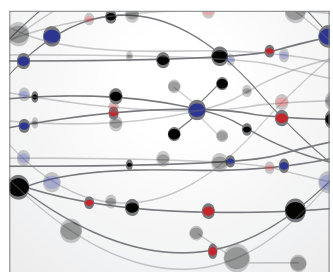

\section{The Scientific} World Journal
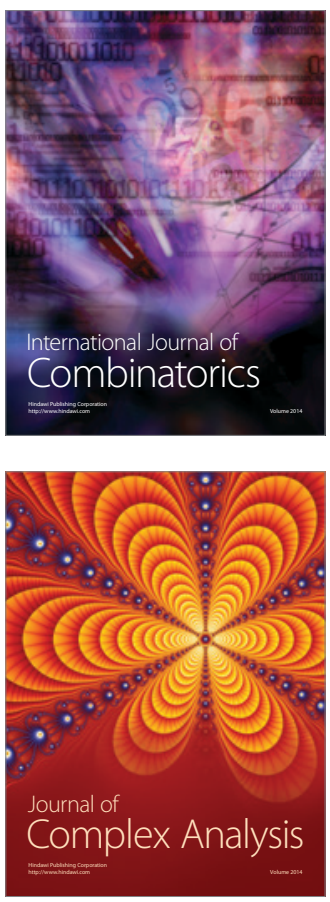

International Journal of

Mathematics and

Mathematical

Sciences
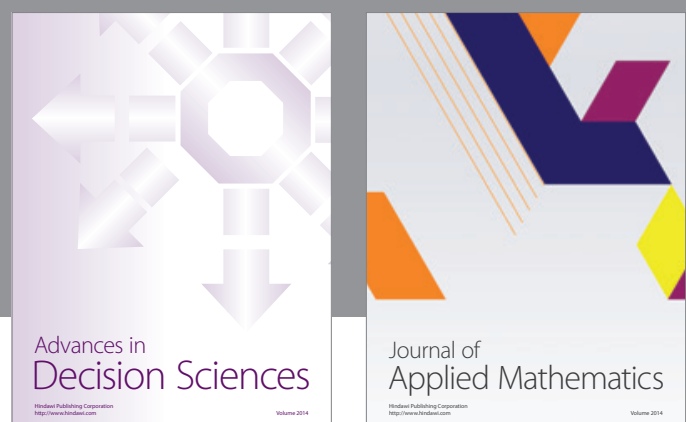

Journal of

Applied Mathematics
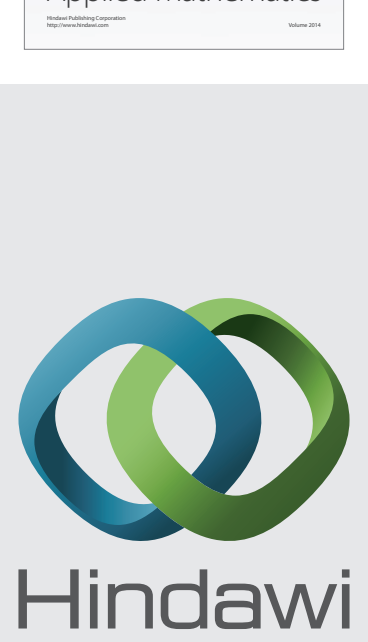

Submit your manuscripts at http://www.hindawi.com
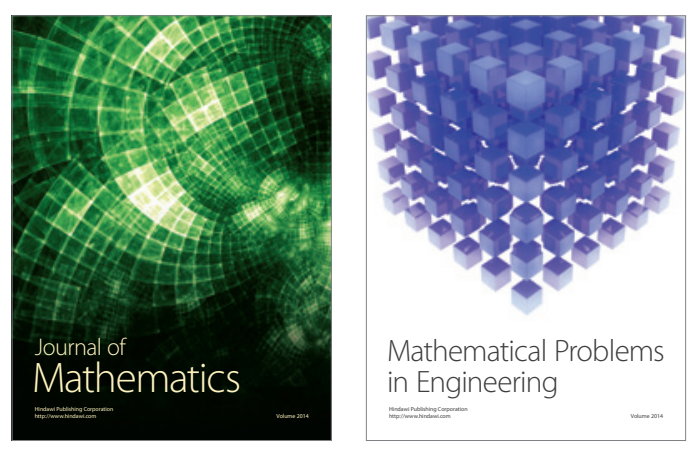

Mathematical Problems in Engineering
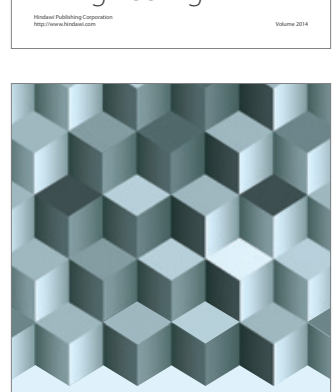

Journal of

Function Spaces
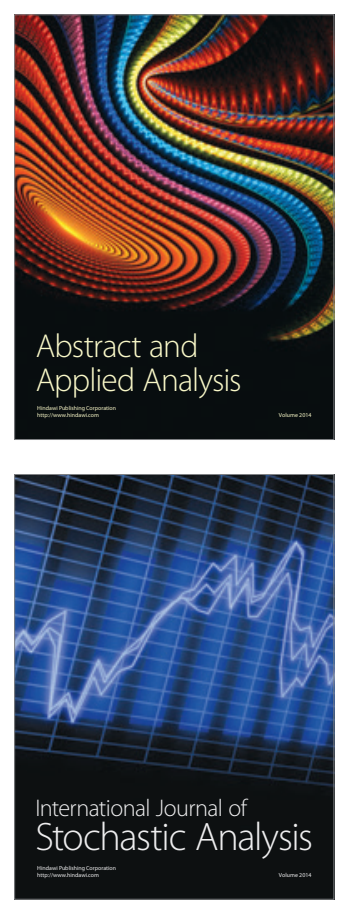

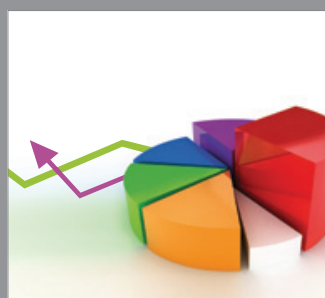

ournal of

Probability and Statistics

Promensencen
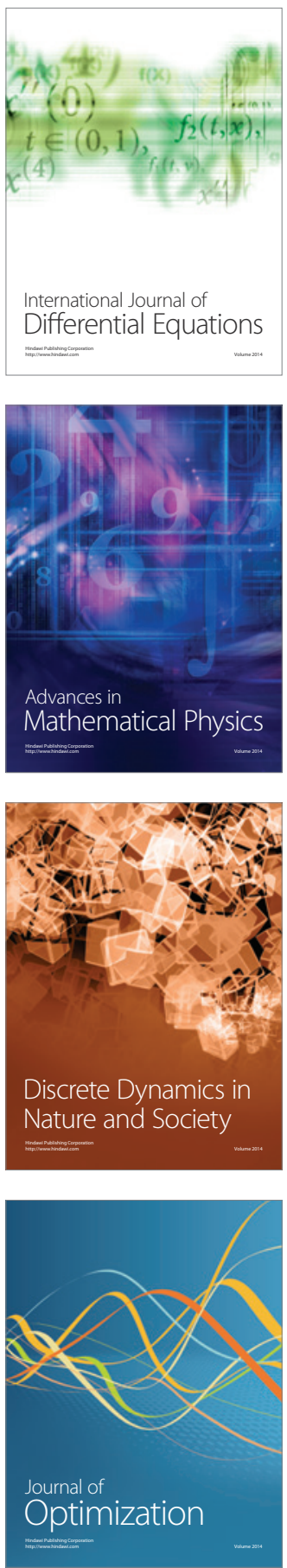